\title{
Unsaturated cell model of effective thermal conductivity of soils
}

\author{
Jianting Zhu' ${ }^{1}$ (1) \\ Received: 7 April 2020 / Accepted: 15 July 2020 / Published online: 17 July 2020 \\ (c) Springer Nature Switzerland AG 2020
}

\begin{abstract}
In this study, a cell model based on new physical conceptualizations is developed to calculate the effective thermal conductivity of unsaturated soils. The evolution of water phase saturation is treated using a cell model consisting of solid particle being enveloped by a mixture of water and air phases. The thermal conductivity of the mixed fluid phase is determined by the volume-weighted average of water and air thermal conductivities. The temperature distribution in the cell of soil and fluid mixture is solved along with boundary condition of linearly varying temperature field. The heat transfer through the cell is calculated by integration of the temperature gradient along with thermal conductivities of soil and fluid. The effective thermal conductivity of the unsaturated soils is determined by conceptualizing the soils as having the same heat transfer as the cell of solid particle and fluid mixture. The new model is compared with 268 data entries of unsaturated soil effective thermal conductivity measurements from the literature. The developed effective thermal conductivity model compares favorably with the experimental data. When the porosity is small, the increase in the effective thermal conductivity with water phase saturation is more significant and non-linear. When the porosity increases, the increases of effective thermal conductivity with water phase saturation approaches a linear relationship.
\end{abstract}

Keywords Effective thermal conductivity · Unsaturated soil · Cell model · Thermal conductivity ratio · Porosity

\section{Introduction}

Soil thermal conductivity is a critical parameter in many environmental and engineering applications, such as ground heat exchangers, ground heat storage, building foundations, pipe lines, and radioactive waste disposal. It is also useful for the coupling of soil, water, heat, and solute in numerical models. However, experiments to measure soil thermal conductivity are usually labor intensive and time-consuming. Therefore, there is a pressing need for soil thermal conductivity models that use basic soil characteristics for predictions. Many models of effective properties such as thermal conductivity and permittivity of multiphase materials have been developed. A recent review was given by Dong et al. [8]. These models can be roughly classified into three major categories [26] of empirical equations [5], mixing models $[3,5]$ including the simple arithmetic mean and geometric and harmonic mean models, and analytical solutions [9].

Various empirical equations have been proposed to relate the effective property to the volume fractions of the multiple phase components by fitting the experimental data. Several empirical models $[4,6,12,15]$ demonstrated good performance, but generally underestimated the thermal conductivity of sands. Two-phase mixing models have been extended to multiple phases, such as parallel, series, and ideally mixed equations for estimating the effective thermal conductivity of multiphase materials [3, 13]. Another type of the mixing models was to treat two of the phases as one single phase with their own effective properties and then to mix this effective phase with another new phase. This type of models usually requires introducing additional empirical parameters [7]. Friedman [9] proposed an analytical model for the unsaturated

Jianting Zhu, jzhu5@uwyo.edu | 'Department of Civil and Architectural Engineering, University of Wyoming, Laramie, WY 82071, USA. 
porous medium effective property by assuming that solid, water, and air phases form a mixture of composite spheres with the radial order of the concentric shells of each phase depending on its saturation degree. Miyamoto et al. [17] extended Friedman's model into four layers and illustrated that no single model was able to give reasonable predictions of thermal conductivity for the three-phase materials. Gori and Corasaniti [10] developed a cubic cell model for the thermal conductivity of three-phase porous media in which water was considered to either cover the solid particles or form liquid-bridges between different particles. However, the critical water content for the liquidbridge formation had to be estimated empirically.

More recently, Tarnawski and Leong [22] proposed an improved geometric mean model for the effective thermal conductivity of unsaturated soils with three soil structurebased parameters. He et al. [11] developed an exponential type model with two fitting parameters and an equation to calculate dry soil thermal conductivity. Bi et al. [2] developed a three-parameter model of thermal conductivity at full range of saturation degree for fine-grained soils based on the normalized thermal conductivity approach. Zhang et al. [27] proposed a matrix of 16 calculation models to examine the effects of dried-soil thermal conductivity models and unsaturated-soil effective thermal conductivity models on the calculation results of soil thermal conductivities. The matrix of 16 calculation models was based on the combinations of 1 saturated-soil thermal conductivity model, 4 dried-soil thermal conductivity models and 4 effective thermal conductivity models for unsaturated soils. The model matrix was evaluated by 40 Canadian soil samples and the results demonstrated that the effective thermal conductivity models have stronger effect on the calculation results of soil thermal conductivity than the dried-soil thermal conductivity models. Ren et al. [19] evaluated the performance of ten normalized models of soil thermal conductivity using published datasets and analyzed the advantages and disadvantages of each model. Wang et al. [25] developed a thermal conductivity model of geomaterials in terms of volumetric fractions of each component and shape factors for unfrozen and freezing states. The model was evaluated and found to match well against experimental data.

Experimental data of unsaturated-soil thermal conductivity that include measurements of all relevant variables/parameters are scarce. Singh et al. [21] measured the effective thermal conductivity of air, water, ice and tertiary amyl alcohol saturated sand systems by a transient method using a line heat source and the known power supplied to the probe heater. By using the transient hot wire method, Kohout et al. [13] measured the effective thermal conductivity of mono- and poly-dispersed random assemblies of both spherical particles and irregular crystals. Based on the results, the effect of volume fractions and spatial distributions of the vapor, liquid, and solid phases on the effective thermal conductivity was investigated. Tarnawski et al. [24] measured the thermal conductivities of 40 field soil samples in Canada using a non-stationary probe technique. The thermal conductivity measurements were conducted over an entire range of saturation from dryness to full saturation. The measured results demonstrated a strong nonlinear variation of the soil thermal conductivity against water phase saturation. McCombie et al. [16] developed an experimental procedure to determine the thermo-physical properties of a coarse soil in Italy over a full range of saturation degree at two porosities based on the transient thermal probe approach. The measured thermal conductivity results revealed an increasing trend with decreasing porosity and increasing water saturation.

As discussed earlier, most unsaturated soil thermal conductivity models are empirical or semi-empirical involving fitting parameters with vague or no clear physical meaning. The objective of this study is to develop a new cell model based on novel physical conceptualizations to estimate thermal conductivity of unsaturated porous materials in which all parameters have clear physical meaning. The cell model conceptualizes the unsaturated porous medium as solid particle being surrounded by a mixture of liquid and gas phases. The evolution of liquid phase saturation is taken into account by the change of liquid phase volume fraction in the liquid-gas mixture, whose thermal conductivity is determined by the volume-weighted mean of liquid and gas thermal conductivities. The developed model is then evaluated against 268 data entries of unsaturated soil effective thermal conductivity measurements from the literature. The model is also compared with the simple mixing models in the form of arithmetic mean and geometric mean of multiple phase thermal conductivities, which do not involve fitting empirical parameters either. Based on the developed model, the effect of liquid phase saturation, porosity, and thermal conductivity ratios on the effective thermal conductivity of unsaturated porous materials is then quantitatively analyzed and discussed.

\section{Methods}

\subsection{Mixed fluid phase thermal conductivity}

In the proposed cell model, the fluid phase effective thermal conductivity consisting of liquid and gas in general (i.e., water and air in unsaturated soils particular) is first determined as the volume-weighted average of water and air thermal conductivities: 
$k_{f}=S_{l} k_{l}+\left(1-S_{l}\right) k_{g}$

where $k_{f}$ is the fluid phase thermal conductivity, $k_{l}$ and $k_{g}$ are the thermal conductivities of liquid and gas, respectively, and $S_{\text {I }}$ denotes the water (liquid) phase saturation. A more general equation to estimate fluid phase thermal conductivity has also been developed $[14,20]$ as follow:

$k_{f}=S_{l}^{m} k_{l}+\left(1-S_{l}^{m}\right) k_{g}$

where $\mathrm{m}$ is the second Archie exponent in the range of 1.5-2.5 [1]. The Archie exponent describes the relation of water phase tortuosity with saturation. In this study, a simple form of volume-weighted average is used (i.e., $m=1$ ). The effect of water phase saturation on the fluid phase thermal conductivity is incorporated in the cell model as described below.

\subsection{Cell model of effective thermal conductivity of unsaturated porous medium}

The proposed cell model is conceptually similar to the model of thermal conductivity in two-phase saturated porous media recently developed by Zhu [28]. The fluid phase is now a mixture of water and air (or liquid and gas in general). The radius of the solid particle at the center of the cell, $R_{s}$, represents the average radius of the solid particles in the unsaturated porous materials (Fig. 1). The radius of the outer mixed fluid envelope, $R_{f}$ is uniquely related to the porosity of the unsaturated porous materials, $n$, to ensure that the cell and the porous material have the same porosity:

$\left(R_{f} / R_{s}\right)^{3}=(1-n)^{-1}$

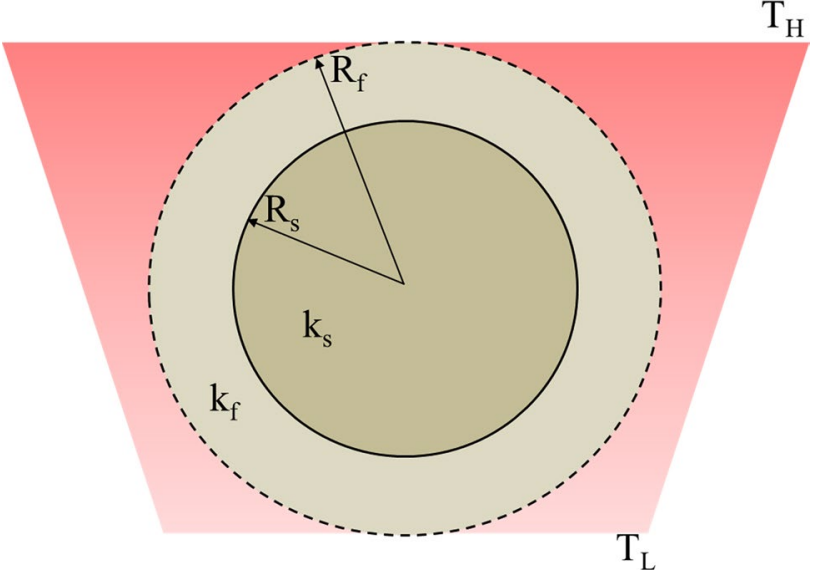

Fig. 1 Diagram of the cell model of heat conduction in unsaturated medium to represent interactions among phases
While $R_{f}$ is the radius of fluid surrounding the solid with the thermal conductivity being determined from Eq. (3), the radius of water shell (or liquid in general) can be determined from the water (liquid) phase saturation, $S_{1}$, by the following equation:

$\frac{R_{l}}{R_{s}}=\left(\frac{n S_{l}}{1-n}+1\right)^{1 / 3}$

Equation (4) can be used to describe how water (liquid) phase radius surrounding the solid evolves with the increase or decrease in the water (liquid) phase saturation.

The cell of soil and fluid mixture undergoes the same given temperature gradient of $\nabla T=\frac{T_{H}-T_{L}}{2 R_{f}}$, shown schematically in Fig. 1. This temperature gradient is the same as the unsaturated porous materials with the effective thermal conductivity. The boundary condition on the outer surface of the cell has the temperature field varying linearly in the direction of heat conduction to ensure that the overall temperature gradient over the cell is $\nabla T$. Based on the given boundary condition, the temperature distribution inside the cell can be analytically determined by solving the Laplace's equation. The total volumetric heat flux through the cell including both solid and fluid phases can then be determined by integrating the temperature gradient in both the solid and fluid phases. The expression for the total volumetric heat flux is similar to the equation in [28] as expressed in Eq. (5) below. However, the radius $R_{f}$ and thermal conductivity $k_{f}$ of fluid shell in Eq. (5) need to be the effective fluid phase properties as determined in Eq. (1) earlier.

$$
\begin{aligned}
Q & =-4 \pi k_{f} C_{f} R_{s}^{3}\left\{\frac{1}{3}\left[\left(\frac{R_{f}}{R_{s}}\right)^{3}-1\right]\right. \\
& \left.+2\left(\frac{k_{s} / k_{f}-1}{k_{s} / k_{f}+2}\right) \ln \left(\frac{R_{f}}{R_{s}}\right)\right\}-\frac{4}{3} \pi k_{s} C_{s} R_{s}^{3}
\end{aligned}
$$

where $Q$ is the total heat flux through the cell, $k_{f}$ is the mixed fluid phase thermal conductivity determined in Eq. (1), and $\mathrm{k}_{\mathrm{s}}$ is the thermal conductivity of solid phase. The coefficients $C_{f}$ and $C_{s}$ in Eq. (5) can be determined by the following equations [28]:

$$
\begin{aligned}
& C_{f}=\frac{\left(k_{s} / k_{f}+2\right) \nabla T}{k_{s} / k_{f}+2+\left(1-k_{s} / k_{f}\right)\left(\frac{R_{s}}{R_{f}}\right)^{3}} \\
& C_{s}=\frac{3 \nabla T}{k_{s} / k_{f}+2+\left(1-k_{s} / k_{f}\right)\left(\frac{R_{s}}{R_{f}}\right)^{3}}
\end{aligned}
$$

Using the effective porous medium idea, the effective thermal conductivity can be determined to ensure that 
the effective porous medium would conduct the same total volumetric heat flux as the cell given in Eq. (5):

$Q=-k_{e} \frac{4}{3} \pi R_{f}^{3} \nabla T$

where $k_{e}$ is the effective thermal conductivity of the porous materials.

From Eqs. (5) and (8), the effective thermal conductivity of the unsaturated porous materials can be determined as:
In the following section, the cell model expressed in Eq. (11) is compared with 268 data entries of unsaturated effective thermal conductivity measurements from the literature along the arithmetic and geometric mean models expressed in Eqs. (12) and (13). For comparison with the experimental data, three statistical indices of root mean square error (RMSE), the linear correlation coefficients ( $\rho)$, and Nash-Sutcliffe model efficiency coefficient (NSE) [18] are used to quantitatively measure the ability of models to

$\frac{k_{e}}{k_{f}}=\frac{\left(\frac{R_{s}}{R_{f}}\right)^{3}\left\{\left(k_{s} / k_{f}+2\right)\left[\left(\frac{R_{f}}{R_{s}}\right)^{3}-1\right]+6\left(k_{s} / k_{f}-1\right) \ln \left(\frac{R_{f}}{R_{s}}\right)+3\left(k_{s} / k_{f}\right)\right\}}{k_{s} / k_{f}+2-\left(k_{s} / k_{f}-1\right)\left(\frac{R_{s}}{R_{f}}\right)^{3}}$

Expressed in terms of the porosity n, Eq. (9) can also be written as:

$\frac{k_{e}}{k_{f}}=\frac{3 k_{s} / k_{f}-2\left(k_{s} / k_{f}-1\right)[n+(1-n) \ln (1-n)]}{3+n\left(k_{s} / k_{f}-1\right)}$ predict the experimental data. Then the impact of liquid phase saturation, porosity, and thermal conductivity ratios of the three phases on the effective thermal conductivity of porous materials is discussed.

Equation (10) can also be expressed as the ratio of $k_{e}$ over water (liquid) phase thermal conductivity as follows:

\section{Results and discussion}

$\frac{k_{e}}{k_{l}}=\frac{\left\{3\left(\frac{k_{s}}{k_{l}}\right)-2\left[\frac{k_{s}}{k_{l}}-S_{l}-\left(1-S_{l}\right)\left(\frac{k_{g}}{k_{l}}\right)\right][n+(1-n) \ln (1-n)]\right\}\left[S_{l}+\left(1-S_{l}\right) \frac{k_{g}}{k_{l}}\right]}{(3-n)\left[S_{l}+\left(1-S_{l}\right)\left(\frac{k_{g}}{k_{l}}\right)\right]+n\left(\frac{k_{s}}{k_{l}}\right)}$

To use the developed model (11), the required input includes only the thermal conductivities of all the three phases involved, $\mathrm{k}_{\mathrm{s}}, \mathrm{k}_{1}$, and $\mathrm{k}_{\mathrm{g}}$, the porosity of the porous materials, $n$, and the degree of water (liquid) phase saturation, $\mathrm{S}_{\text {. }}$. Since all the parameters in the developed model (11) have clear physical meaning, it will be compared with two convenient models often used to estimate effective thermal conductivity with the same required input parameters (i.e., arithmetic mean and geometric mean of volume-weighted thermal conductivities of the three phases). For conductors in parallel, the effective thermal conductivity is the volume-weighted arithmetic mean, which is the theoretical upper bound for the effective thermal conductivity [13]:

$k_{\text {arith }}=(1-n) k_{s}+S_{l} n k_{l}+\left(1-S_{l}\right) n k_{g}$

The effective thermal conductivity of ideally mixed phase is their volume-weighted geometric mean as follows [13]:

$k_{\text {geo }}=k_{s}^{1-n} k_{l}^{S_{l} n} k_{g}^{\left(1-s_{l}\right) n}$
While there have been numerous models developed over the last few decades as discussed in the Introduction section earlier, most models include empirical and/or semi-empirical parameters with vague or no clear physical meaning. It is beyond the scope of this study to comprehensively review these models and compare them with the present model. Instead, the developed effective thermal conductivity model is compared and validated with experimental data in the literature along with the arithmetic mean and geometric mean models.

While the solid phase thermal conductivity $k_{s}$ in Eq. (11) has a clear physical meaning, it is, however, sensitive to soil mineral composition $[16,24]$ and represents a lumped thermal conductivity of actual soil minerals. Therefore, it is not easy to be directly measured since soil mineralogy is not commonly measured in practice. Most of the experimental results of effective thermal conductivity measurements in the literature did not include the $k_{s}$ values. One approach to estimate $k_{s}$ is a combined use of experimental saturated thermal conductivity data and the weighted geometric mean model $[16,23]$. To facilitate comparison with geometric mean and arithmetic mean models in relation to experimental results, the required solid phase 
thermal conductivity $k_{s}$ is inversely estimated from the reported experimental results of water saturated porous medium thermal conductivity $k_{\text {sat }}$ if it was not given in the original literature. Quantitatively, the $k_{s}$ value for each sample is determined from Eqs. (11), (12), and (13) with $\mathrm{S}_{\mathrm{I}}=1$ and $\mathrm{k}_{\mathrm{e}}=\mathrm{k}_{\mathrm{sat}}$ for the present model (Eq. 14), arithmetic mean model (Eq. 15), and geometric mean model (Eq. 16), respectively, as follows:

$k_{s}=\frac{k_{\text {sat }}(3-n)-2[n+(1-n) \ln (1-n)] k_{l}}{3-2[n+(1-n) \ln (1-n)]-n\left(\frac{k_{\text {sat }}}{k_{l}}\right)}$

$k_{s}=\frac{k_{\text {sat }}-n k_{l}}{1-n}$

$k_{s}=\left(\frac{k_{s a t}}{k_{l}^{n}}\right)^{\frac{1}{1-n}}$

Figure 2 shows the results of comparison with a comprehensive experimental data set of effective thermal conductivity of 40 soil samples in Canada from the study of Tarnawski et al. [24] at various saturations. The estimated $\mathrm{k}_{\mathrm{s}}$ values for the 40 soil samples are in the range from 1.50 to $8.47 \mathrm{Wm}^{-1} \mathrm{~K}^{-1}, 1.67$ to $4.62 \mathrm{Wm}^{-1} \mathrm{~K}^{-1}$ and 2.15 to 8.03 $\mathrm{Wm}^{-1} \mathrm{~K}^{-1}$ for the present model, the arithmetic mean model and the geometric mean model, respectively, which are close to those estimated based on the geometric mean model by Tarnawski et al. [24]. The water and air thermal

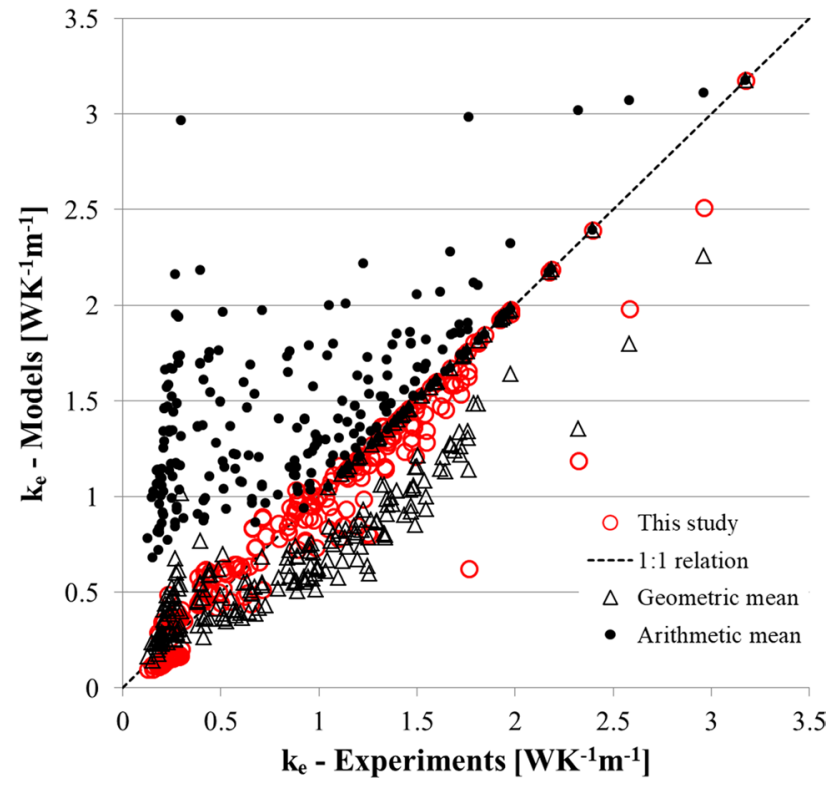

Fig. 2 Comparison with the experimental data from Tarnawski et al. [24]. The predictions from the geometric mean and arithmetic mean models are also included for comparison conductivities are $0.61 \mathrm{Wm}^{-1} \mathrm{~K}^{-1}$ and $0.026 \mathrm{Wm}^{-1} \mathrm{~K}^{-1}$, respectively. There were 240 experimental data-point entries for the 40 soil samples. The RMSEs of the present model, the geometric mean model and arithmetic mean model are $0.159 \mathrm{Wm}^{-1} \mathrm{~K}^{-1}, 0.288 \mathrm{Wm}^{-1} \mathrm{~K}^{-1}$ and 0.701 $\mathrm{Wm}^{-1} \mathrm{~K}^{-1}$, respectively. The linear correlation coefficients for the three models are $0.968,0.915$ and 0.642 respectively. The NSEs of the three models are $0.932,0.778$ and -0.318 , respectively. These statistical index values demonstrate that the present model is significantly better than the geometric mean model and arithmetic mean model. From Fig. 2, it can be seen that the present model significantly under-estimates only one of the 40 soil samples. This under-estimated sample is a Sable sand from Nova Scotia (code NS-04), which was clearly an outlier exhibiting much higher thermal conductivity over the entire range of water phase saturation compared to all other samples. In general, higher values of thermal conductivity were obtained in soil samples with high quartz content from Nova Scotia and Prince Edward Island. Further quantitative analysis indicated that the NS-04 sample had thermal conductivity that was $20.6 \%, 192 \%, 100 \%, 62.8 \%, 57.1 \%$ and $47.8 \%$ higher than the average thermal conductivities of the other 6 samples from Nova Scotia at $S_{1}=0.0,0.1,0.25$, $0.5,0.7$, and 1.0 , respectively. Therefore, the NS-04 sample not only had significantly higher thermal conductivity than the other samples but also increased the fastest with increasing water phase saturation in the low water saturation range compared to the similar soil samples from the same region.

Figure 3 shows the comparison with a more recent experimental study [16] with 12 data points at various water saturations, in which a transient thermal probe method was used to measure the thermal conductivity over an entire range of the saturation degree at two porosities of 0.44 and 0.50 . The water and air thermal conductivities are given as $0.61 \mathrm{Wm}^{-1} \mathrm{~K}^{-1}$ and 0.026 $\mathrm{Wm}^{-1} \mathrm{~K}^{-1}$, respectively. The RMSEs of the present model, the geometric mean model and arithmetic mean model are determined to be $0.073 \mathrm{Wm}^{-1} \mathrm{~K}^{-1}, 0.172 \mathrm{Wm}^{-1} \mathrm{~K}^{-1}$ and $0.429 \mathrm{Wm}^{-1} \mathrm{~K}^{-1}$, respectively. The linear correlation coefficients for the three models are 0.984, 0.919 and 0.932, respectively. The NSEs of the three models are $0.959,0.767$ and -0.447 , respectively. Therefore, the results also demonstrate the proposed model performs much better than the geometric mean model and arithmetic mean model.

Figure 4 shows the comparison results with the experimental study of Kohout et al. [13] of 16 data points at various saturations, which were measured using the transient hot wire method. The solid materials are four size classes of glass beads with a thermal conductivity of $0.8 \mathrm{Wm}^{-1} \mathrm{~K}^{-1}$, mean diameters of $68 \mu \mathrm{m}, 112 \mu \mathrm{m}, 200 \mu \mathrm{m}$, and $628 \mu \mathrm{m}$, respectively. Deionised water was used as the liquid with 


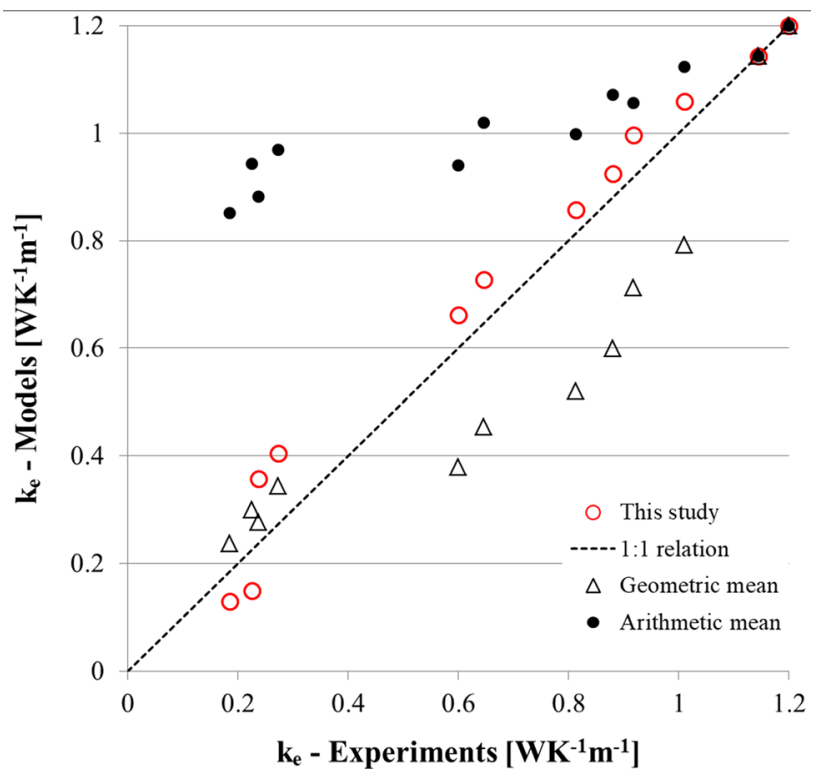

Fig. 3 Comparison with the experimental data from McCombie et al. [16]. The predictions from the geometric mean and arithmetic mean models are also included for comparison

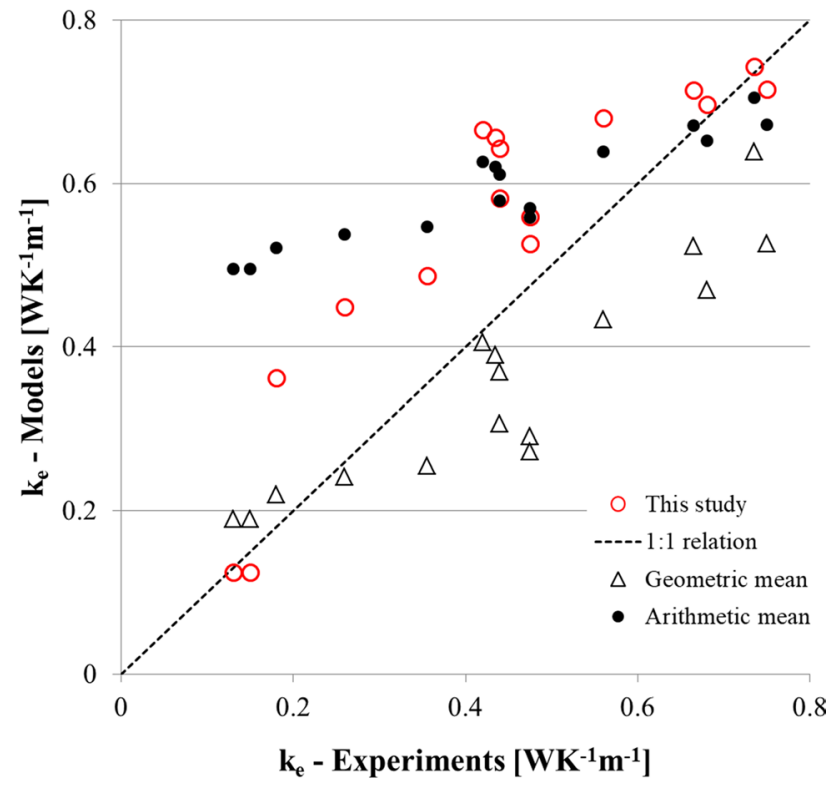

Fig. 4 Comparison with the experimental data from Kohout et al. [13]. The predictions from the geometric mean and arithmetic mean models are also included for comparison

a thermal conductivity of $0.61 \mathrm{Wm}^{-1} \mathrm{~K}^{-1}$. The air thermal conductivity is $0.02 \mathrm{Wm}^{-1} \mathrm{~K}^{-1}$. The RMSEs of the present model, the geometric mean model and arithmetic mean model are $0.134 \mathrm{Wm}^{-1} \mathrm{~K}^{-1}, 0.127 \mathrm{Wm}^{-1} \mathrm{~K}^{-1}$, and 0.200 $\mathrm{Wm}^{-1} \mathrm{~K}^{-1}$, respectively. The linear correlation coefficients for the three models are $0.889,0.918$, and 0.938 respectively. The NSEs of the three models are $0.520,0.568$, and
-0.073 respectively. These statistical index values are not as good as those for the soil samples shown in Figs. 2 and 3. In general, the present model and arithmetic mean model over-estimate the experimental results while the geometric mean model under-estimate them. Given that water thermal conductivity is significantly higher than the air thermal conductivity, the effective thermal conductivity of the unsaturated soils increases with the increase in the water phase saturation. However, it should be noticed that there were significant scatters in the experimental data. It should be noted that the solid in the experimental data in Fig. 4 was glass beds with much lower thermal conductivity than the soil grains in Figs. 2 and 3, which explain the main reason that the experimental thermal conductivity values in Fig. 4 were smaller than those in Figs. 2 and 3. From Fig. 2, it can be observed that the present model tends to over-estimate the experimental thermal conductivities in the small range of less than $1.0 \mathrm{Wm}^{-1} \mathrm{~K}^{-1}$ for the samples with small water phase saturation. For those samples, the water phase could be discontinuous due to its low saturation. The present model, however, assumes that the water phase is uniformly mixed with air phase and the mixed fluid phase envelopes the solid grains. This assumption could exaggerate the role of water phase and overpredict the experimental thermal conductivities in situations when the water phase is discontinuous.

The developed model that takes into account the evolution of water phase saturation is generally able to capture the effective thermal conductivity relationship with water phase saturation. As shown in Fig. 5a, the liquid
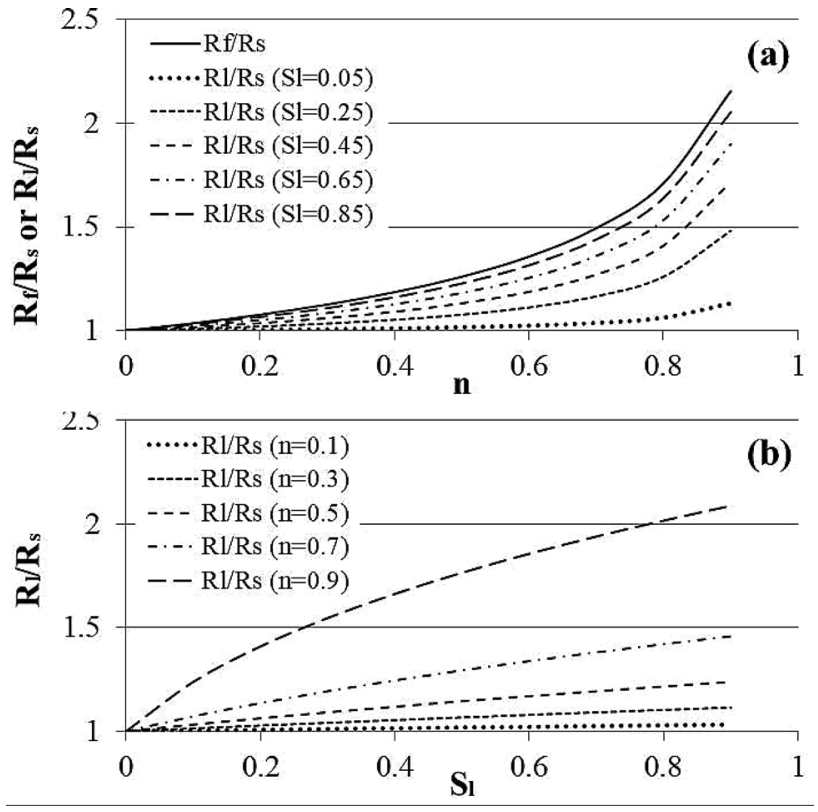

Fig. 5 Fluid phase radius evolution with a porosity $n$, and $\mathbf{b}$ liquid phase saturation, $\mathrm{S}_{1}$, in the proposed cell model 
phase radius in the cell model increases first slowly with the porosity, which means with small increase in the liquid volume, the liquid cell tends to concentrate near the solid and magnify the impact of liquid phase on the effective thermal conductivity. This would create the convex relationship between the $k_{e}$ and $S_{\text {, }}$ which is both predicted by the present model and observed in most experimental results $[4,16,24]$. The arithmetic mean model predicts a linear relationship while the geometric mean model predicts a concave relation. The more significant impact of $S_{1}$ in its low range on the effective thermal conductivity can also be explained by examining how liquid phase cell radius increases with the liquid phase saturation, $S_{1}$, which is a convex relationship as shown in Fig. $5 \mathrm{~b}$. In the present model, the evolution of liquid phase saturation in relation to the solid phase is more physically represented, and as a result, the developed model better captures the evolution of effective thermal conductivity with liquid phase saturation. The cell model reflects the fact that as water phase saturation increases, the rate of liquid phase radius increase slows down as the curve becomes flat shown in Fig. 5b. Compared to the simple models of geometric mean and arithmetic mean, the cell model can better capture the convex relationship between the effective thermal conductivity and liquid phase saturation.

Figure 6 shows the ratio of the effective thermal conductivity over the liquid phase conductivity $k_{e} / k_{1}$ as a function of liquid phase saturation when the ratio of solid over liquid thermal conductivities $k_{s} / k_{1}$ is 5 and the ratio of gas over liquid thermal conductivities $k_{g} / k_{1}$ is 0.1 . When the porosity $\mathrm{n}$ is larger, the impact of solid becomes smaller,

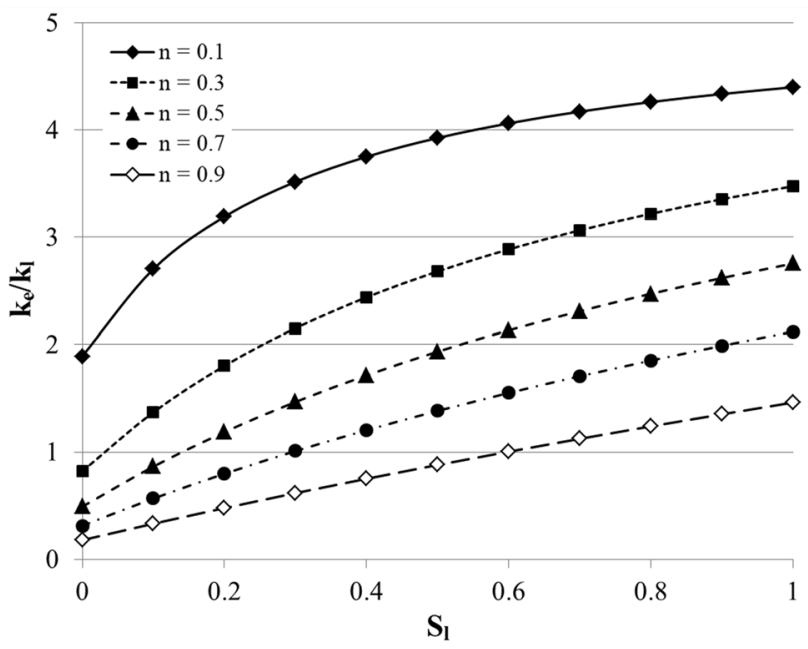

Fig. 6 The ratio of effective thermal conductivity over the liquid phase conductivity $\left(k_{e} / k_{l}\right)$ as a function of liquid phase saturation $\left(S_{1}\right)$ when the ratio of solid over liquid thermal conductivities $\left(k_{s} / k_{1}\right)$ is 5 and the ratio of gas over liquid thermal conductivities $\left(k_{g} / k_{1}\right)$ is 0.1 which means $k_{e}$ is also smaller since $k_{s}$ is higher than $k_{1}$. When the porosity $n$ is small, the increase in $k_{e}$ with $S_{l}$ is more significant at low range of $\mathrm{S}_{\text {, }}$ which captures the general convex relation of $k_{e}$ increase in relation to $S_{1}$ in experimental data. When the porosity $\mathrm{n}$ increases, the increases in $\mathrm{k}_{\mathrm{e}}$ with $\mathrm{S}_{1}$ approaches a linear relationship.

Figure 7 plots the ratio of effective thermal conductivity over the liquid phase conductivity $k_{e} / k_{1}$ as a function of the ratio of solid over liquid thermal conductivities $k_{s} /$ $\mathrm{k}_{1}$ when the liquid saturation $\mathrm{S}_{\text {, }}$ is 0.5 and the ratio of gas over liquid thermal conductivities $k_{g} / k_{1}$ is 0.1 . When the porosity $n$ is small, $k_{e}$ increases dramatically with $k_{s} / k_{1}$ since $\mathrm{k}_{\mathrm{s}}$ dictates $\mathrm{k}_{\mathrm{e}}$ when there is small amount of fluid in the porous materials. However, the influence of fluid on the effective thermal conductivity is still very significant even at small porosity.

Figure 8 shows the results of the ratio of effective thermal conductivity over the liquid phase conductivity $k_{e} / k_{1}$ as a function of porosity $n$ when the liquid saturation $S_{1}$ is 0.5 and the ratio of gas over liquid thermal conductivities $\mathrm{k}_{\mathrm{g}} / \mathrm{k}_{\mathrm{l}}$ is 0.1 . When $\mathrm{k}_{\mathrm{s}} / \mathrm{k}_{\mathrm{l}}$ is larger than 1 (i.e., solid is more conductive than liquid, which is the case for most porous systems), the effective thermal conductivity decreases non-linearly from the solid thermal conductivity when $\mathrm{n}=0$ to the fluid phase thermal conductivity when $\mathrm{n}=1$. On the other hand, when $k_{s} / k_{1}$ is smaller than 1 , the effective thermal conductivity increases non-linearly from the solid thermal conductivity when $n=0$ to the fluid phase thermal conductivity when $n=1$.

While our focus in this study is on the effective thermal conductivity of unsaturated soils, the approach can be used for other porous materials, including estimating

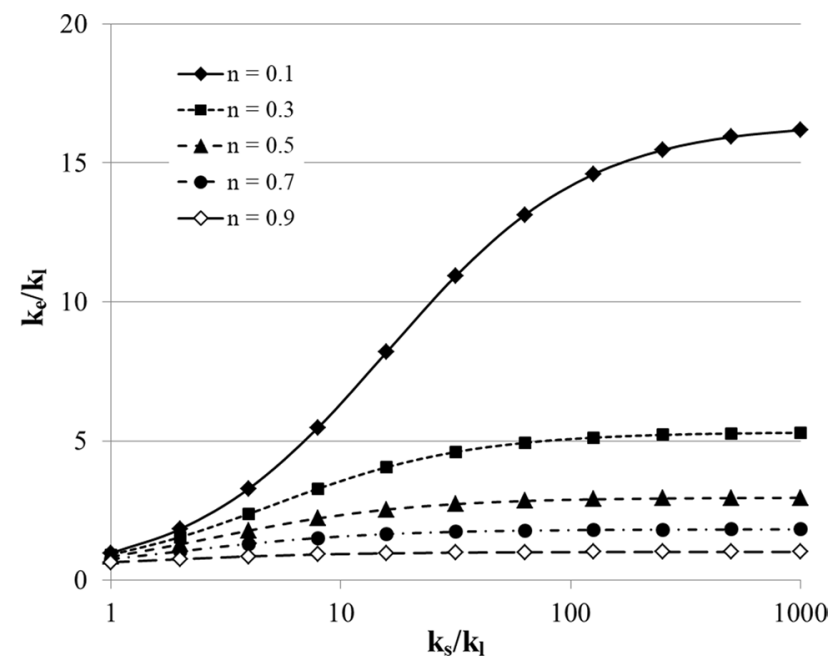

Fig. 7 The ratio of effective thermal conductivity over the liquid phase conductivity $\left(k_{e} / k_{1}\right)$ as a function of the ratio of solid over liquid thermal conductivities $\left(k_{s} / k_{1}\right)$ when liquid saturation $\left(S_{1}\right)$ is 0.5 and the ratio of gas over liquid thermal conductivities $\left(k_{g} / k_{1}\right)$ is 0.1 


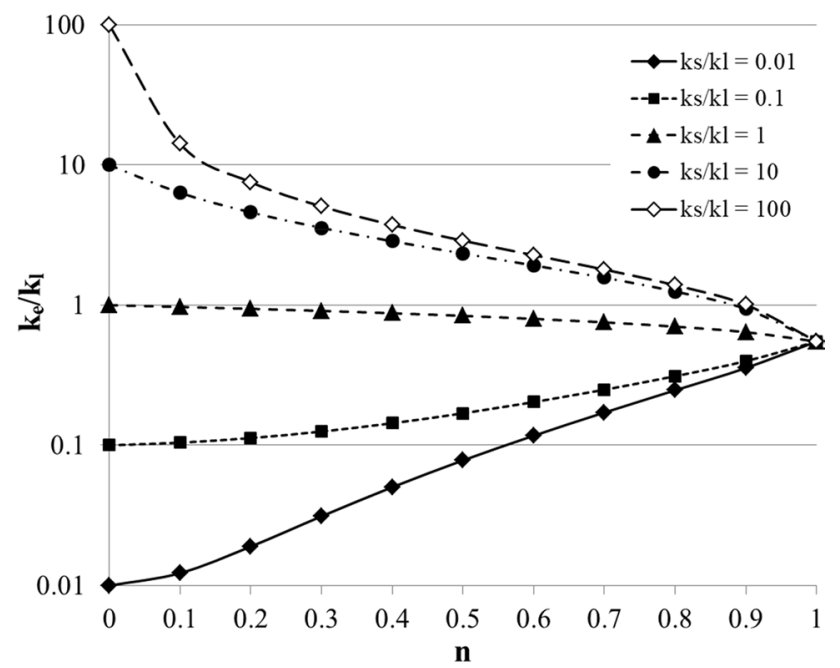

Fig. 8 The ratio of effective thermal conductivity over the liquid phase conductivity $\left(k_{e} / k_{l}\right)$ as a function of porosity $(n)$ when liquid saturation $\left(\mathrm{S}_{\mathrm{l}}\right)$ is 0.5 and the ratio of gas over liquid thermal conductivities $\left(k_{g} / k_{l}\right)$ is 0.1

temporal evolution of effective thermal conductivity for processes involving role of water phase such as ceramic processing where the microstructure changes in relation to water phase over time. The model first lumps the two fluid phases together and determines their effective thermal conductivity as the simple volume-weighted mean of their respective thermal conductivity. The cell model idea then only applies to one mixed fluid phase and the solid phase, which is similar to the mixing model concept. This model can be improved by considering the three-phase cell model and solving heat conduction equation separately for each individual phase.

\section{Conclusions}

In this study, a new cell model is developed to determine the effective thermal conductivity of unsaturated porous materials. The thermal conductivity of the mixed fluid phases is determined by the volume-weighted mean of gas and liquid thermal conductivities. The effective thermal conductivity of the unsaturated porous materials is then determined by conceptualizing the unsaturated porous materials as having the same heat transfer as the cell of solid particle and fluid. The new model is compared with 268 data entries of unsaturated effective thermal conductivity measurements from the literature. The main conclusions are summarized as follows.

1. The developed effective thermal conductivity model compares favorably with the experimental data in the literature given that all parameters in the model have clear physical meaning.

2. The model over-estimates the experimental data mainly in situations where solid and water phases have small contrast in their thermal conductivity or in situations where water saturation is low.

3. When $n$ is small, the increase in $k_{e}$ with $S_{l}$ is more significant at small range of $\mathrm{S}_{\text {. }}$. When the porosity increases, the increases in $\mathrm{k}_{\mathrm{e}}$ with $\mathrm{S}_{\mathrm{I}}$ approaches a linear relationship.

4. When $n$ is small, $k_{e}$ increases dramatically with $k_{s} / k_{1}$ since $k_{s}$ dictates $k_{e}$ when there is small amount of fluid in the porous systems. However, the influence of fluid on the effective thermal conductivity is still very significant even at small porosity.

5. When $k_{s} / k_{1}$ is larger than 1 , the effective thermal conductivity decreases non-linearly from the solid thermal conductivity for $n=0$ to the fluid thermal conductivity for $n=1$. On the other hand, the effective thermal conductivity increases non-linearly from the solid thermal conductivity for $\mathrm{n}=0$ to the fluid thermal conductivity for $n=1$ when $k_{s} / k_{l}$ is smaller than 1 .

\section{Compliance with ethical standards}

Conflict of interest The author declares no conflict of interest.

\section{References}

1. Archie GE (1942) The electrical resistivity log as an aid in determining some reservoir characteristics. Trans Am Inst Min Metall Eng 146:54-62. https://doi.org/10.2118/942054-G

2. Bi J, Zhang M, Chen W, Lu J, Lai Y (2018) A new model to determine the thermal conductivity of fine-grained soils. Int J Heat Mass Transf 123:407-417. https://doi.org/10.1016/j.ijheatmass transfer.2018.02.035

3. Chandrakanthi M, Mehrotra AK, Hettiaratchi JPA (2005) Thermal conductivity of leaf compost used in biofilters: an experimental and theoretical investigation. Environ Pollut 136:167-174. https://doi.org/10.1016/j.envpol.2004.09.027

4. Chen S (2008) Thermal conductivity of sands. Heat Mass Transf 44:1241-1246. https://doi.org/10.1007/s00231-007-0357-1

5. Cosenza P, Guerin R, Tabbagh A (2003) Relationship between thermal conductivity and watercontent of soils using numerical modelling. Eur J Soil Sci 54:81-587. https://doi.org/10.104 6/j.1365-2389.2003.00539.x

6. Cote J, Konrad JM (2005) A generalized thermal conductivity model for soils and construction materials. Can Geotech J 42:443-458. https://doi.org/10.1139/t04-106

7. Dobson MC, Ulaby FT, Hallikainen MT, Elrayes MA (1985) Microwave dielectric behavior of wet soil-part II: dielectric mixing models. IEEE Trans Geosci Remote Sens 23:35-46. https ://doi.org/10.1109/TGRS.1985.289498 
8. Dong Y, McCartney JS, Lu N (2015) Critical review of thermal conductivity models for unsaturated soils. Geotech Geol Eng 33:207-221. https://doi.org/10.1007/s10706-015-9843-2

9. Friedman SP (1998) A saturation degree-dependent composite spheres model for describing the effective dielectric constant of unsaturated porous media. Water Resour Res 34:2949-2961. https://doi.org/10.1029/98WR01923

10. Gori F, Corasaniti S (2003) Experimental measurements and theoretical prediction of the thermal conductivity of two- and three-phase water/olivine systems. Int J Thermophys 24:13391353. https://doi.org/10.1023/A:1026107319415

11. He H, Zhao Y, Dyck MF, Si B, Jin H, Lv J, Wang J (2017) A modified normalized model for predicting effective soil thermal conductivity. Acta Geotech 12:1281-1300. https://doi.org/10.1007/ s11440-017-0563-z

12. Jougnot $D$, Revil A (2010) Thermal conductivity of unsaturated clay-rocks. Hydrol Earth Syst Sci 14:91-98. https://doi. org/10.5194/hess-14-91-2010

13. Kohout M, Collier AP, Stepanek F (2004) Effective thermal conductivity of wet particle assemblies. Int J Heat Mass Transf 47:5565-5574. https://doi.org/10.1016/j.ijheatmasstrans fer.2004.07.031

14. Linde N, Binley A, Tryggvason A, Pedersen LB, Revil A (2006) Improved hydrogeophysical characterization using joint inversion of cross-hole electrical resistance and ground penetrating radar travel time data. Water Resour Res 42:W12404. https://doi. org/10.1029/2006WR005131

15. Lu S, Ren T, Gong Y, Horton R (2007) An improved model for predicting soil thermal conductivity from water content at room temperature. Soil Sci Soc Am J 71:8-14. https://doi.org/10.2136/ sssaj2006.0041

16. McCombie ML, Tarnawski VR, Bovesecchi G, Coppa P, Leong WH (2017) Thermal conductivity of pyroclastic soil (Pozzolana) from the environs of Rome. Int J Thermophys 38:21. https://doi. org/10.1007/s10765-016-2161-y

17. Miyamoto T, Annaka T, Chikushi J (2005) Extended dual composite sphere model for determining dielectric permittivity of andisols. Soil Sci Soc Am J 69:23-29. https://doi.org/10.2136/ sssaj2005.0023

18. Nash JE, Sutcliffe JV (1970) River flow forecasting through conceptual models part I: a discussion of principles. J Hydrol 10:282-290. https://doi.org/10.1016/0022-1694(70)90255-6
19. Ren J, Men L, Zhang W, Yang J (2019) A new empirical model for the estimation of soil thermal conductivity. Environ Earth Sci 78:361. https://doi.org/10.1007/s12665-019-8360-7

20. Revil A, Linde N, Cerepi A, Jougnot D, Matthai S, Finsterle $S$ (2007) Electrokinetic coupling in unsaturated porous media. J Colloid Interface Sci 313:315-327. https://doi.org/10.1016/j. jcis.2007.03.037

21. Singh AK, Singh R, Chaudhary DR (1990) Prediction of effective thermal conductivity of moist porous materials. J Phys D Appl Phys 23:698-702. https://doi.org/10.1088/0022-3727/23/6/010

22. Tarnawski VR, Leong WH (2016) Advanced geometric mean model for predicting thermal conductivity of unsaturated soils. Int J Thermophys 37:18. https://doi.org/10.1007/s1076 5-015-2024-y

23. Tarnawski VR, Momose T, Leong WH (2011) Thermal conductivity of standard Sands II. Saturated conditions. Int J Thermophys 32:984-1005. https://doi.org/10.1007/s10765-011-0975-1

24. Tarnawski VR, Momose T, McCombie ML, Leong WH (2015) Canadian field soils III. Thermal-conductivity data and modeling. Int J Thermophys 36:119-156. https://doi.org/10.1007/s1076 5-014-1793-z

25. Wang C, Lai Y, Zhang M, Li S (2019) A generalized thermal conductivity model of geomaterials based on micro-structures. Acta Geotech 14:1423-1436. https://doi.org/10.1007/s1144 0-018-0728-4

26. Wang M, Pan N (2008) Predictions of effective physical properties of complex multiphase materials. Mater Sci Eng R Rep 63:1-30. https://doi.org/10.1016/j.mser.2008.07.001

27. Zhang M, Bi J, Chen W, Zhang X, Lu J (2018) Evaluation of calculation models for the thermal conductivity of soils. Int Commun Heat Mass Transf 94:14-23. https://doi.org/10.1016/j.icheatmass transfer.2018.02.005

28. Zhu J (2019) A cell model of effective thermal conductivity for saturated porous media. Int J Heat Mass Transf 138:1054-1060. https://doi.org/10.1016/j.ijheatmasstransfer.2019.04.134

Publisher's Note Springer Nature remains neutral with regard to jurisdictional claims in published maps and institutional affiliations. 